This is a self-archived - parallel published version of this article in the publication archive of the University of Vaasa. It might differ from the original.

\title{
Robust Primary Control of Microgrids for Parametric and Topological Uncertainties: A Quest for Resilience
}

Author(s): Baghaee, Hamid Reza; Parizad, Ali; Shafie-Khah, Miadreza; Siano, Pierluigi; Senatore, Adolfo

Title: Robust Primary Control of Microgrids for Parametric and Topological Uncertainties: A Quest for Resilience

Year: $\quad 2019$

Version: Accepted manuscript

Copyright (C)2019 IEEE. Personal use of this material is permitted. Permission from IEEE must be obtained for all other uses, in any current or future media, including reprinting/republishing this material for advertising or promotional purposes, creating new collective works, for resale or redistribution to servers or lists, or reuse of any copyrighted component of this work in other works.

Please cite the original version:

Baghaee, H. R., Parizad, A., Shafie-Khah, M., Siano, P. \& Senatore, A. (2019). Robust Primary Control of Microgrids for Parametric and Topological Uncertainties: A Quest for Resilience. In: 2019 23rd International Conference on Mechatronics Technology (ICMT), 1-5. https://doi.org/10.1109/ICMECT.2019.8932113 


\section{Robust Primary Control of Microgrids for Parametric and Topological Uncertainties: A Quest for Resilience}

\author{
Hamid Reza Baghaee \\ Dept. Electrical Engineering \\ Amirkabir University of \\ Technology, \\ Tehran, Iran \\ hrbaghaee@aut.ac.ir
}

\author{
Ali Parizad \\ Dept. Electrical \\ Engineering, Southern \\ Illinois University \\ Carbondale, IL, USA \\ ali.parizad@ieee.org
}

\author{
Miadreza Shafie-khah \\ School of technology and \\ Innovations \\ University of Vaasa \\ Vaasa, Finland \\ mshafiek@univaasa.fi
}

\author{
Pierluigi Siano \\ Dept. Management \& \\ Innovation Systems \\ University of Salerno \\ Fisciano (SA), Italy \\ psiano@unisa.it
}

\author{
Adolfo Senatore \\ Dept. Industrial \\ Engineering \\ University of Salerno \\ Fisciano (SA), Italy \\ a.senatore@unisa.it
}

\begin{abstract}
In this paper, a robust droop-based control structure is developed in primary level of a hierarchical control scheme for robust performance and robust stability against parametric and topological uncertainties to 1) improve the robust stability and robust performance 2 ) resolve drawbacks of previously reported methods. Considering droop control scheme, the conventional hierarchical control structure is developed and the effectiveness of the proposed control scheme is investigated considering parametric and topological uncertainties. For the sake of drawing a picture to address how we can realize a resilient microgrid including a hierarchical control structure and providing enough robustness against mentioned uncertainties, signal disturbances, and different types of nonlinearity, some special recommendations are also provided. Finally, to prove the usefulness of the proposed controller, simulation studies are done on a microgrid which includes several distributed generation units with local loads.
\end{abstract}

Keywords - Distributed energy resources, droop control, hierarchical control, microgrid, power sharing, primary control, robust control, stability, uncertainties.

\section{INTRODUCTION}

The coexistence of multiple distributed/renewable energy resources (DER/RERs) units with different dynamic features have raised several concerns such as control, stability and efficiency of microgrids. Based on this issue, there are some concerns reported in microgrid resiliency such as 1) How to share power, 2) How to consider communication time-delay (CTD) and fault ride through (FRT), 3) How one can include the impacts of nonlinear/unbalanced loads in the microgrid power-flow methods [1]-[2].

Typically, the converter related to voltage sources (VSC) in the based microgrids can be managed essentially based on two main methods namely centralized and decentralized control. Centralized techniques have been proposed for limited applications and demand excessive communications [1]. Decentralized control works on the basis of local measurement of important variables and transferring them to the relevant local controllers (LC) that is not communicate to other LCs. hierarchical control scheme is divided into three levels, i.e., primary, secondary and tertiary [3]. In an autonomous microgrid, the control objectives are restoration of frequency and voltage in order to share active/reactive power, properly [3]-[5]. In grid-connected modes, voltage and frequency are based on the upstream network and the basic objectives are controlling active and reactive power [6].
In both modes, the microgrid performance may be affected by different parametric, topological, and communication uncertainties, different nonlinearities (either by nonlinear loads or dynamics of DERs/RERs) and various small- and large-signal events/disturbances. To have a resilient microgrid, the system should have robust performance during all of the uncertainties, nonlinearities, and disturbances [7]. Some new solutions have been proposed to address robust stability of microgrid for signal disturbances [8], in case of nonlinear/unbalanced loads as well as DERs dynamics. Control of microgrid and robust power flow considering different issues under topological, parametric, and communication system uncertainty (in addition to noise and communication time-delay (CTD)) have been discussed in [5], [8]-[11].

In this paper, a robust hierarchical drop-based control structure is reported to resolve issues regarding small- and large-signal stability of microgrid, improve the performance of microgrid for nonlinear and unbalanced loads, and provide robust performance for parametric and topological uncertainties of microgrid. We propose a robust controller for the inner control loops of primary control level in the hierarchical control scheme and effectively can resolve the stability issues for various kinds of disturbances. Moreover, the robust performance of the proposed robust controller for parametric and topological is considered in the design formulation. Finally, the performance of this control strategy is evaluated by simulations performed in MATLAB/Simulink environment for outage and re-connection of DER units, as a topological uncertainty which leads to a large-signal disturbance, and examines the plug and play functionality of microgrids.

\section{STUDY SYSTEM}

\section{A. Proposed Control Strategy Configuration}

Fig.1 shows the block diagram of the proposed robust primary controller which is a part of an improved robust hierarchical control. Our goal is to design $K(s)$ so that we can improve the stability, minimize the consequence of disturbance on the output voltage, and accomplish to a good performance taking the impact of CTD into consideration. We propose a mixed $H_{2} / H_{\infty}$ solution to realize the design objectives mentioned in section I to be utilized in the control loop of primary level (in this case inner loop) to track the reference 


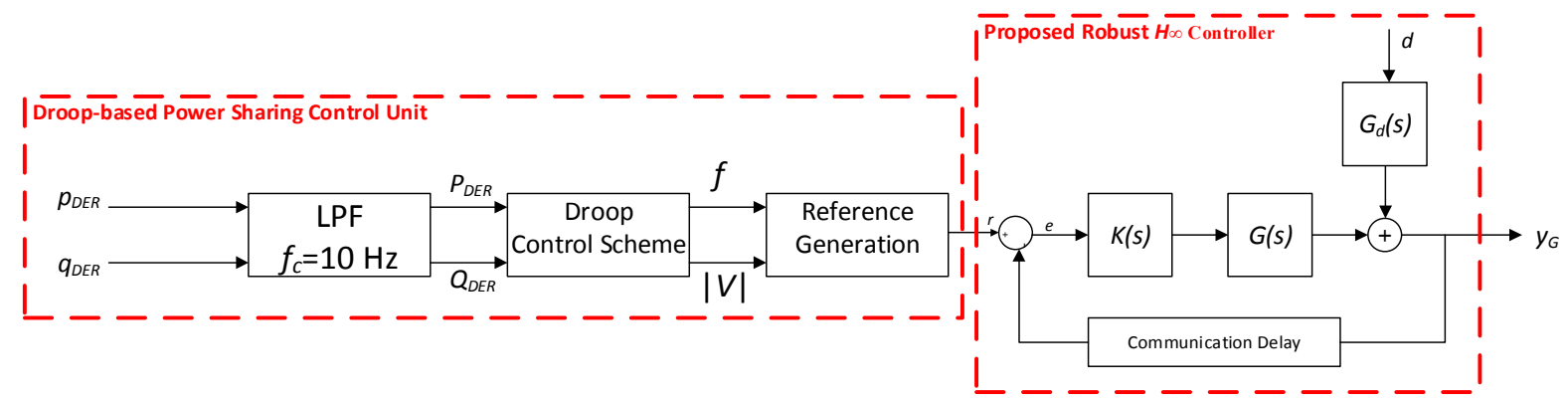

Fig. 1. Proposed robust controller configuration in the inner control loops of primary control level of hierarchical control.

values and improve the microgrid stability. In contrast to the DRSM controller of [5], this configuration will also help the overcurrent and overload protection [12].

\section{B. Power Management System}

Space equations for the system are referred to $\square \square$ reference frame applying Clarke transformation [13]. The immediate active/reactive power of each DER unit and load is transferred to the PMS and LCs via an LBC system. Active/ reactive powers $\left(P_{D E R, I,} Q_{D E R, i}\right)$ and voltage setpoints of DER can be calculated by:

$$
\begin{aligned}
& P_{D E R, i}=\sum_{j=1}^{N}\left|V_{i}\right|\left|V_{j}\right|\left|Y_{i j}\right| \cos \left(\delta_{i}-\delta_{k}-\theta_{i j}\right) \\
& Q_{D E R, i}=\sum_{j=1}^{N}\left|V_{i}\right|\left|V_{j}\right|\left|Y_{i j}\right| \sin \left(\delta_{i}-\delta_{k}-\theta_{i j}\right)
\end{aligned}
$$

\section{MiCROGRID MATHEMATICAL MODEL}

Fig. 2 shows the proposed robust controller after linearization of microgrid model in the SYRF frame $(d q)$ [13].

In our study, the load current is considered as disturbance signal. Moreover, dynamic equations of microgrid are expressed in NARF frame $(a b c)$ and then referend to SYRF. Based on this issue, the realization known as state space realization for the microgrid is given by:

$$
\begin{aligned}
& x=A x+B u+B_{d} d \\
& y=C x
\end{aligned}
$$

where,

$$
\begin{aligned}
x^{T}= & \left(V_{1 d}, V_{1 q}, I_{1 d}, I_{1 q}, I_{t 1 d}, I_{t 1 q},\right. \\
& V_{2 d}, V_{2 q}, I_{2 d}, I_{2 q}, I_{t 2 d}, I_{t 2 q}, \\
& \left.V_{3 d}, V_{3 q}, I_{3 d}, I_{3 q}\right), \\
u^{T}= & \left(V_{t 1 d}, V_{t 1 q}, V_{t 2 d}, V_{t 2 q}, V_{t 3 d}, V_{t 3 q}\right), \\
d^{T}= & \left(I_{L 1 d}, I_{L 1 q}, I_{L 2 d}, I_{L 2 q}, I_{L 3 d}, I_{L 3 q}\right), \\
y^{T}= & \left(V_{1 d}, V_{1 q}, V_{2 d}, V_{2 q}, V_{3 d}, V_{3 q}\right)
\end{aligned}
$$

system state space matrices are defined as [7]:

$$
A \subset \mathfrak{R}^{16 \times 16}, B \subset \mathfrak{R}^{16 \times 6}, B_{d} \subset \mathfrak{R}^{16 \times 6} \text { and } C \subset \mathfrak{R}^{6 \times 16} \text { are }
$$

We try to find a controller $K(s)$ with the state space realization of (5) to realize the design objectives.

$$
K(s):\left\{\begin{array}{l}
\dot{\zeta}=A_{k} \zeta+B_{k} y \\
u=C_{k} \zeta+D_{k} y
\end{array}\right.
$$

\section{Problem Statement}

\section{A. Nominal Problem}

The goal of this study is to design decentralized robust controller for robust stabilization and disturbance attenuation. Moreover, microgrid performance disturbances (small/ large-signal) are investigated. Consistent with this idea, disturbance signal $d$ has some effects on the output $y_{G}$. This effect has been reduced and the output gain $\left(£_{2}\right)$ minimized. In this manner, we have a good reference tracking for controller and therefore the steady state error should be minimized. In the presented robust controller design, disturbance and tracking attenuation are employed as the objective functions and the form of multi-objective problem defined by LMI are applied as constraints. The problem can be formulated in the form of the following multi-objective optimization problem that will result in LMIs given by (7):

$$
\begin{array}{ll}
\min _{K(s)}\left\|W_{d}(I+G K)^{-1} G_{d}\right\|_{\infty}, & \min _{K(s)}\left\|W_{1}(I+G K)^{-1}\right\|_{2} \\
\text { st. } \quad\left\|W_{2} T_{r u}\right\|_{\infty}<1, & \left\|W_{1}(I+G K)^{-1}\right\|_{\infty}<1,
\end{array}
$$

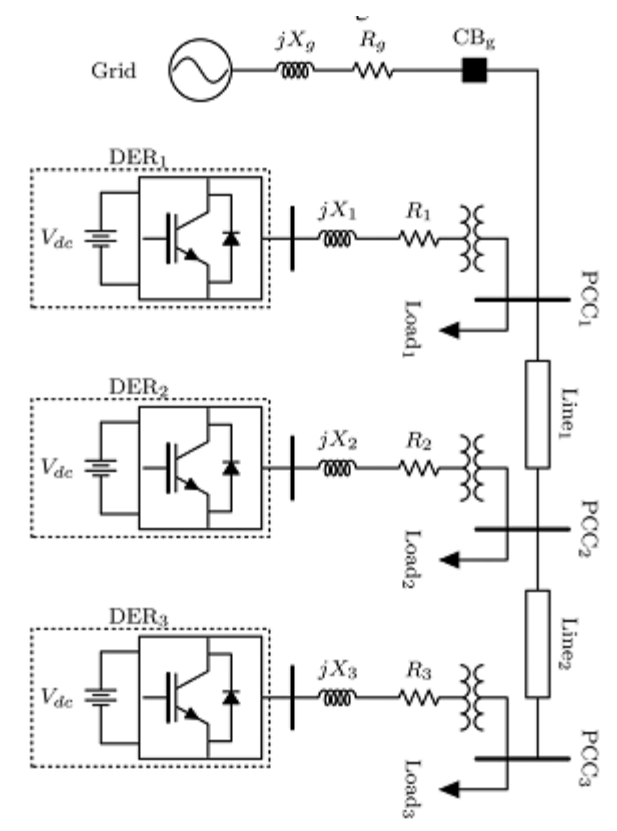

Fig. 2. Schematic diagram of study system [12]. 
In (6), microgrid voltage limits are considered in order to design a system with acceptable parameters.

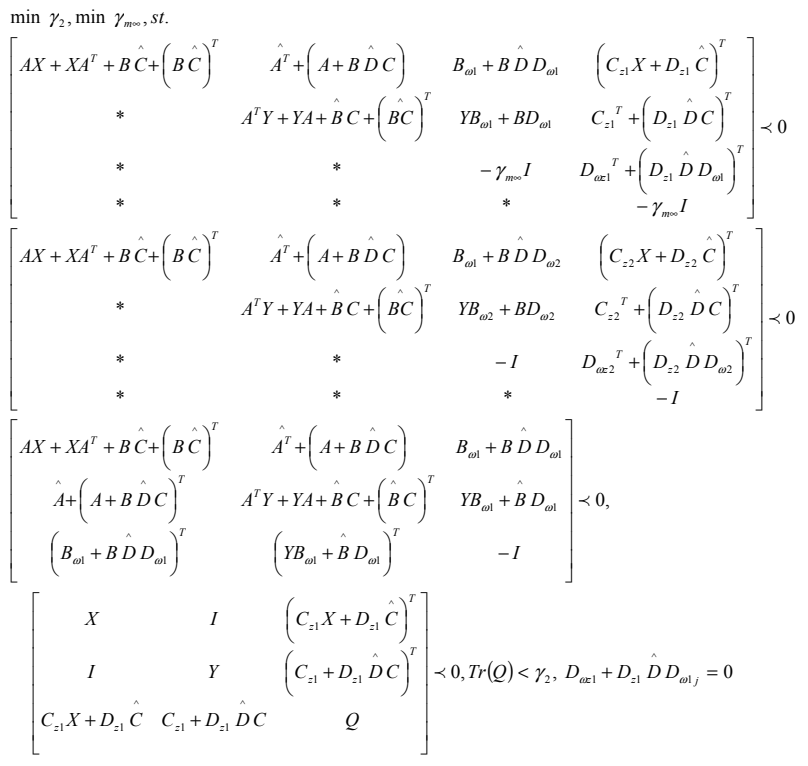

\section{B. Robust Controller Design}

Based on the assumption of a polytope of uncertainties for microgrid parameters, the abovementioned controller design is extended to a robust mixed $H_{2} / H_{\infty}$ problem. More details have been provided in [6], [9].

Also, it is worth mentioning that the PMS determines the initial conditions of the controller set points based on a robust power flow solution provided that will enhance the robust performance of the proposed scheme.

\section{Plug and Play Functionality}

Robustness against microgrid topological uncertainties and keeping the P\&P functionality of DER units is another property that is integrated with the proposed robust control scheme. Since the plug-in (out) of DER $j$ to (from) DER $i$ affects only matrix Aii, for each DG two cases are taken into account [14]:

1) Maximum allowable connections of the DGs to DER $i$

$(N i m a x \subset\{1, \ldots, N\})$

2) Connection $j$ with the minimum values of $\left(R_{i j} / Z_{i j}^{2}\right)$ and $\left(X_{i j} / Z_{i j}^{2}\right)$ among the other.

$$
\left(\frac{R}{Z^{2}}\right)_{\min }=\min _{j \in N_{i}} \frac{R_{i j}}{Z_{i j}^{2}} ;\left(\frac{X}{Z^{2}}\right)_{\min }=\min _{j \in N_{i}} \frac{X_{i j}}{Z_{i j}^{2}}
$$

Matrix Agii for the mentioned cases is as follows:

$$
\begin{aligned}
& A_{g i i}^{1}=\left[\begin{array}{cccc}
-\frac{1}{C_{t i}} \sum_{j \in N_{i, \max }} \frac{R_{i j}}{Z_{i j}^{2}} & \omega_{0}-\frac{1}{C_{t i}} \sum_{j \in N_{i, \max }} \frac{X_{i j}}{Z_{i j}^{2}} & \frac{k_{i}}{C_{t i}} & 0 \\
-\omega_{0}+\frac{1}{C_{t i}} \sum_{j \in N_{t, \max }} \frac{X_{i j}}{Z_{i j}^{2}} & -\frac{1}{C_{t i}} \sum_{j \in N_{t, \max }} \frac{R_{i j}}{Z_{i j}^{2}} & 0 & \frac{k_{i}}{C_{t i}} \\
-\frac{k_{i}}{L_{t i}} & 0 & -\frac{R_{t i}}{L_{t i}} & \omega_{0} \\
0 & -\frac{k_{i}}{L_{t i}} & -\omega_{0} & -\frac{R_{t i}}{L_{t i}}
\end{array}\right] \\
& A_{g i i}^{2}=\left[\begin{array}{cccc}
-\frac{1}{C_{t i}}\left(\frac{R}{Z^{2}}\right)_{\min } & \omega_{0}-\frac{1}{C_{t i}}\left(\frac{X}{Z^{2}}\right)_{\min } & \frac{k_{i}}{C_{t i}} & 0 \\
-\omega_{0}+\frac{1}{C_{t i}}\left(\frac{X}{Z^{2}}\right)_{\min } & -\frac{1}{C_{t i}}\left(\frac{R}{Z^{2}}\right)_{\min } & 0 & \frac{k_{i}}{C_{t i}} \\
-\frac{k_{i}}{L_{t i}} & 0 & -\frac{R_{t i}}{L_{t i}} & \omega_{0} \\
0 & -\frac{k_{i}}{L_{t i}} & -\omega_{0} & -\frac{R_{t i}}{L_{t i}}
\end{array}\right]
\end{aligned}
$$

In this case, different possible connection/disconnection of DERs to DER $i$ matches to the polytypic uncertainty domain:

$$
\hat{A}_{i i}(\lambda)=\lambda \hat{A}_{i i}^{1}+(1-\lambda) \hat{A}_{i i}^{2}
$$

where $0 \leq \lambda \leq 1$ and,

$$
\hat{A}_{g i i}^{1}=\left[\begin{array}{cc}
A_{g i i}^{1} & 0 \\
-C_{g i} & 0
\end{array}\right], \hat{A}_{g i i}^{2}=\left[\begin{array}{cc}
A_{g i i}^{2} & 0 \\
-C_{g i} & 0
\end{array}\right], i=1, \cdots, N .
$$

Now, these matrices can be added to LMI (7) for consideration of P\&P functionality in controller design.

\section{Mixed $\mathrm{H}_{2} / \mathrm{H}_{\infty}$ and proposed control framework}

In order to optimize the overall performance of the system, the matrix $\boldsymbol{K}$ should be chosen so that the optimization problem of (6) is realized. Considering the state space realizations, the mixed $H_{2} / H_{\infty}$ synthesis problem can be defined by the multi objective optimization problem of (7). In this case, a controller $K(s)$ can be designed in order to minimize a compromised criterion for $H_{2} / H_{\infty}$ performance. The problem is LMI and can be solved by MATLAB via YALMIP solver [15]-[16]. The $H_{2} / H_{\infty}$ performance lemmas link the aforementioned optimization control problem to LMI techniques in the form of LMI of equation (7).

\section{Simulation RESUlts}

In this part, four DER units (with a local load) are connected to the external grid via PPC. This microgrid is implemented in MATLAB/Simulink time-domain simulations in offline mode is analyzed. Microgrid structure is shown in Fig. 2. Also, microgrid parameters as well as control system are given in [5].

\section{A. Islanded Mode - Step Load Change}

In the first scenario, microgrid is in islanded mode with local loads is considered. LCs are participated by the proposed mixed $H_{2} / H_{\infty}$ control scheme. An event is defined to increase the load of DER2 at $t=0.8 \mathrm{sec}$ with step $0.8 \mathrm{pu}$. It can be seen that change of the is responded by DER units and voltage magnitude/angle are stable after this event. 
Fig. 3(a) illustrates the active/reactive power sharing curves of the microgrid, to the modulation index of DER2 VSC proposed mixed $H_{2} / H_{\infty}$ control scheme, and DRSM solution of [5]. It is obvious that after the transient, powersharing trends reach the stable value.

\section{B. Parametric Uncertainties}

Power management system (PMS) and the performance of the control in the microgrid can be affected by parametric uncertainties. Robust control design approaches reported in [5] are applied to the microgrid system in order to employ The robustness against microgrid parameter uncertainties. RBFNN-based power flow has been developed to consider the robustness in case we have changed in the load multiplier (LM) and line's R/X ratio, reported in the other methods like Newton-Raphson (NR). In case the R/X ratio and LM vary, power flow is converged and microgrid has a good performance and therefore there is no distortion in current/voltage waveforms. Based on this matter, it is not necessary to adjust protection and control settings. In rare case, when we have low $\mathrm{R} / \mathrm{X}$ ratio in microgrid, harmonic power sharing will not be accurate since the value of impedance is high when we are working with harmonics in the higher order.

\section{Accidental Outage of DER2}

In order to show the operational integrity of the microgrid and robustness of the proposed control scheme, in this section, sudden accidental outage of the DER2 unit is studied. It is assumed that the two other DERs can meet the load demand. Before the outage, $0.18 \mathrm{pu}$ active power is transferred from PCC1 to PCC2 and there is not power transfer from PCC2 to PCC3. At $t=0.5 \mathrm{sec}$, DER2 is disconnected. Fig. 4 shows corresponding transients of microgrid system. As a consequence of disconnection of
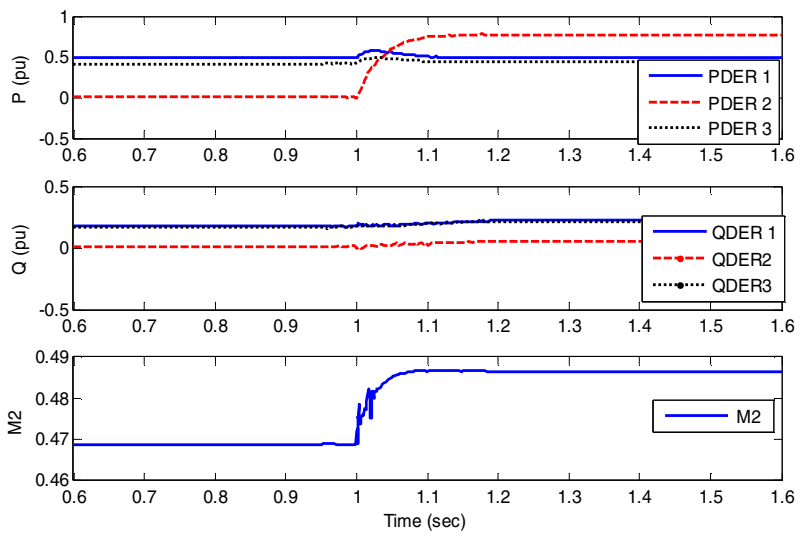

(a)
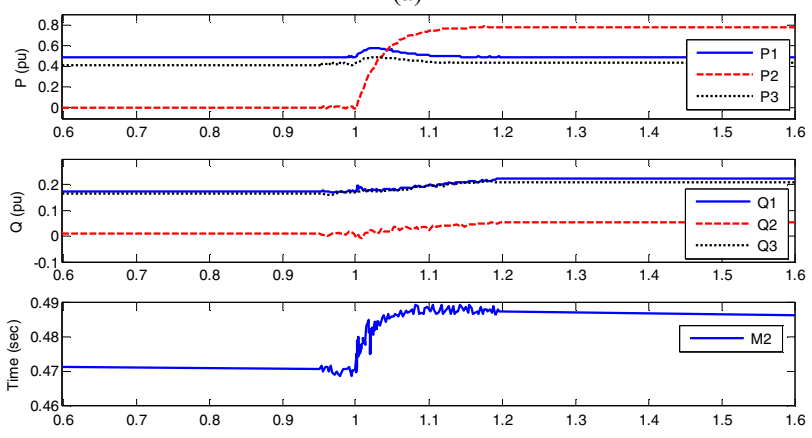

(b)

Fig. 3. Microgrid in the islanded mode (with the the proposed mixed $\mathrm{H} 2 / \mathrm{H} \infty$ robust controller): a) active and reactive power sharing and mudulation index of DER 2 within the microgrid with DRSM solution of [5], b) active/reactive power sharing and mudulation index of DER 2 within the microgrid with proposed mixed $\mathrm{H} 2 / \mathrm{H} \infty$ robust controller.
DER2, the voltage drops by $6 \%$ using the DRSM [5] solution and by only $2 \%$ using the proposed mixed $H_{2} / H_{\infty}$ robust control scheme. Note that in this situation, DER is not directly controlled and voltage of the PCC2 is indirectly controlled by LCs of remaining DER units. Also, as illustrated in Fig. 4, using the proposed mixed $H_{2} / H_{\infty}$ robust controller, the line flows are changed to compensate for the lack of power generation at Bus 2. The results effectively show the robustness of the proposed mixed $H_{2} / H_{\infty}$ robust control scheme in case of deactivation of one of DER units, in contrast with the DRSM solution [5].

\section{CONCLUSION}

In this study, a robust mixed $\mathrm{H} 2 / \mathrm{H} \infty$ controller for a multi-DER VSC-based autonomous microgrid has been presented. A classical load flow has been applied to a microgrid system in order to find 1) terminal voltage set points 2) robust mixed $\mathrm{H} 2 / \mathrm{H} \infty$ controller 3) global synchronization signals 4) frequency control and synchronization of DERs. A set of linear matrix inequalities have been formulated in order to design robust mixed $\mathrm{H} 2 / \mathrm{H} \infty$ control system. Thereafter, multi-objective optimization problem (MOO) was employed to enhance performance of the microgrid for different disturbances, track voltage and active/reactive power setpoints, reject disturbance, and enhance fault ride through capability of the desired power sharing. The effectiveness of proposed method was investigated considering delay in communication line and parametric uncertainties. Offline digital time-domain
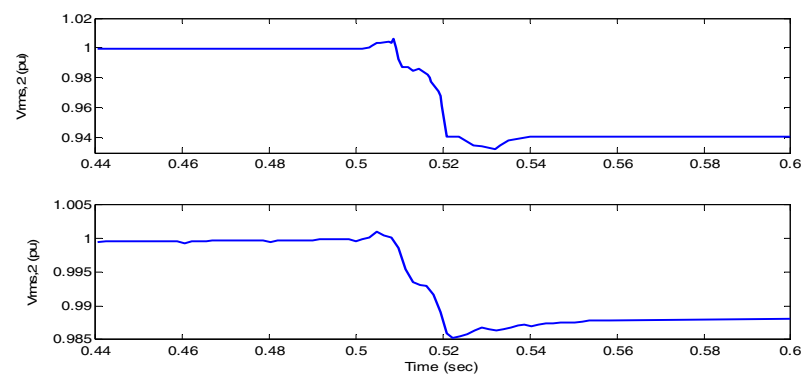

(a)
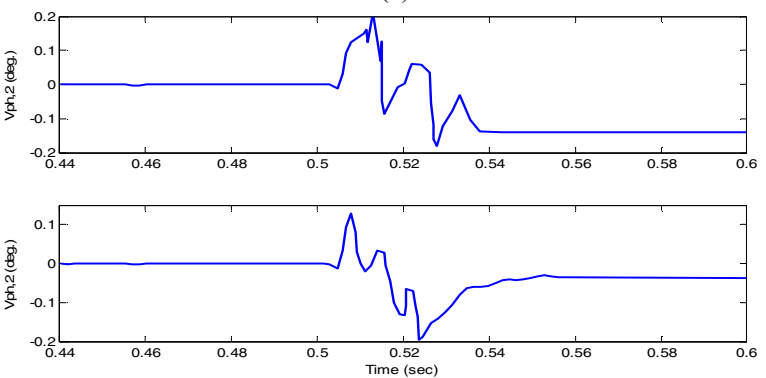

(b)
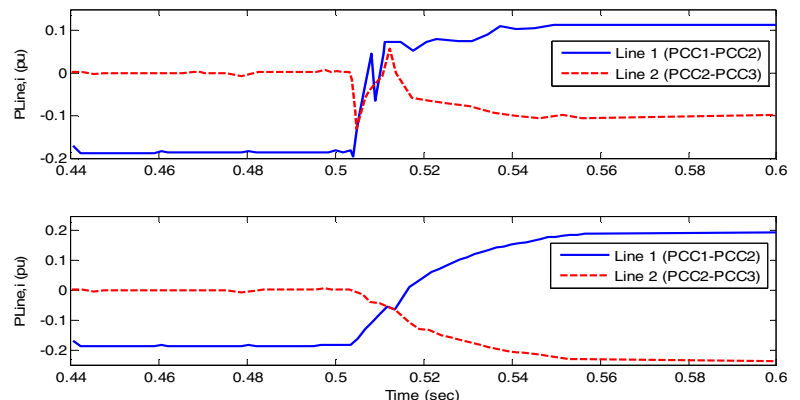

(c)

Fig. 4. Time-domain simulation for accidental outage of DER2 in islanded microgrid for respectively the DRSM solution [5] and, the proposed mixed $\mathrm{H}_{2} / \mathrm{H}_{\infty}$ robust controller: a) voltage magnitude of PCC2 $\left.\left(V_{r m s}\right)(\mathrm{pu}) \mathrm{b}\right)$ phase angle of PCC2 voltage (deg.), c) active power transfer of distribution lines $(\mathrm{pu})$. 
simulations in MATLAB/Simulink show a good robustness against small/large-signal disturbances as well as nonlinearities of load and resource. Results indicated that proposed control strategy keep microgrid stability in an acceptable range and improved its performance after different events such as small/large disturbances.

\section{REFERENCES}

[1] A. Parizad, AH. Khazali, M. Kalantar, "Unbalanced distribution network planning by sitting and sizing of distributed generation and harmonic filter due to losses and THD minimization", Int. Rev. of Elec. Eng., vol. 5, no. 2, part B, pp. 726-736, March -April 2010.

[2] H.R. Baghaee, M. Abedi, "Calculation of weighting factors of static security indices used in contingency ranking of power systems based on fuzzy logic and analytical hierarchy process", International Journal of Electrical Power \& Energy Systems, vol. 33, no. 4, pp. 855-860, 2011.

[3] J.M. Guerrero et. al "Hierarchical control of droop-controlled AC and DC microgrids-A general approach toward standardization", IEEE Trans. Ind. App., vol. 58, no. 1, pp. 158 -172, January 2011.

[4] J.M. Guerrero et al "Advanced control architectur. al.es for intelligent microgrids - part i: decentralized and hierarchical control", IEEE Trans. Ind. App., vol. 60, no. 4, pp. 1254 - 1262, April 2013.

[5] A.H. Etemadi, E.J. Davison, R. Iravani, "A generalized decentralized robust control of islanded microgrids", IEEE Trans. on Power Sys., vol. 29, no. 6, pp. 3102-3113, Oct.2014.

[6] A. Parizad, S. Mohamadian, M. E. Iranian and J. M. Guerrero, "Power System Real-Time Emulation: A Practical Virtual Instrumentation to Complete Electric Power System Modeling," in IEEE Transactions on Industrial Informatics, vol. 15, no. 2, pp. 889900, Feb. 2019.
[7] A. Parizad, H. Khoshkhoo, Sh. Dehghan, and R. Moradtalab. "An intelligent load and generation shedding procedure in an islanded network using a smart power management system." In 2017 Smart Grid Conference (SGC), pp. 1-12. IEEE, 2017.

[8] H. Karimi, E. J. Davison, R. Iravani, "Multivariable servomechanism controller for autonomous operation of a distributed generation unit: Design and performance evaluation," IEEE Trans. Power Syst., vol. 25,no. 2, pp. 853-865, May 2010.

[9] Z. Li, M. Shahidehpour, F. Aminifar, A. Alabdulwahab, Y. Al-Turki, "Networked microgrids for enhancing the power system resilience", Proceedings of the IEEE, vol. 105, no. 7, pp. 1289-13010, July 2017.

[10] M. S. Sadabadi, A. Karimi, H. Karimi, "Fixed-order decentralized/ distributed control of islanded inverter-interfaced microgrids," Control Engineering Practice, vol. 45, pp. 174-193, December 2015.

[11] X. Lu, X. Yu, J. Lai, J.M. Guerrero, H. Zhou, "Distributed secondary voltage and frequency control for islanded microgrids with uncertain communication links", IEEE Trans. on Ind. informatics, vol. 13, no. 2, pp. 448-460, April 2017.

[12] A.H. Etemadi, R. Iravani, "Overcurrent and Overload Protection of Directly Voltage-Controlled Distributed Resources in a Microgrid", IEEE Trans. Ind. Elect., vol. 60, no. 12, pp. 5629 - 5638, June 2013.

[13] J. Lai, H. Zhou, X. Lu, X. Yu, W. Hu, "Droop-based distributed cooperative control for microgrids with time-varying delays", IEEE Trans. Smart Grid, vol. 7, no. 4, pp. 1775-1789, July 2016.

[14] P. Krause, O. Wasynczuk, S. Sudhoff, Analysis of Electric Machinery and Drive Systems, 2nd ed. Hoboken, NJ: Wiley, 2002.

[15] M.S. Sadabadi, Q. Shafiee, A. Karimi, "Plug-and-play voltage stabilization in inverter-interfaced microgrids via a robust control strategy," IEEE Trans. Cont. Syst. Technol., vol. 25, no. 3, pp. 781791, May 2017.

[16] P. Gahinet, A. Nemirovski, A.J. Laub, M. Chilali. LMI control toolbox, South Natick: The MathWorks Inc.; 2006. 